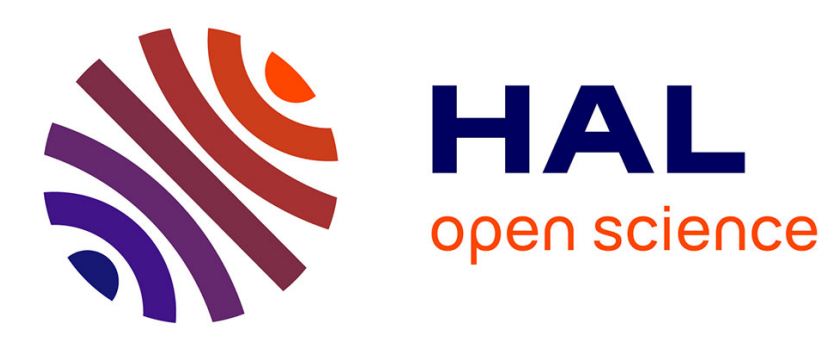

\title{
m-dissipativity of Kolmogorov operators corresponding to Burgers equations with space-time white noise
}

Giuseppe da Prato, Arnaud Debussche

\section{To cite this version:}

Giuseppe da Prato, Arnaud Debussche. m-dissipativity of Kolmogorov operators corresponding to Burgers equations with space-time white noise. Potential Analysis, 2007, 26 (1), pp.31-55. 10.1007/s11118-006-9021-5 . hal-00001354

\section{HAL Id: hal-00001354 https://hal.science/hal-00001354}

Submitted on 25 Mar 2004

HAL is a multi-disciplinary open access archive for the deposit and dissemination of scientific research documents, whether they are published or not. The documents may come from teaching and research institutions in France or abroad, or from public or private research centers.
L'archive ouverte pluridisciplinaire HAL, est destinée au dépôt et à la diffusion de documents scientifiques de niveau recherche, publiés ou non, émanant des établissements d'enseignement et de recherche français ou étrangers, des laboratoires publics ou privés. 


\title{
$m$-dissipativity of Kolmogorov operators corresponding to Burgers equations with space-time white noise
}

\author{
Giuseppe Da Prato \\ Scuola Normale Superiore, 56126, Pisa, Italy \\ daprato@sns.it \\ Arnaud Debussche \\ Ecole Normale Supérieure de Cachan, antenne de Bretagne \\ Campus de Ker Lann, 35170 Bruz, France \\ debussche@bretagne.ens-cachan.fr
}

\begin{abstract}
In this article, we prove that the Kolmogorov operator associated to the Burgers equation driven by a space-time white noise is $m$-dissipative. This implies several properties on the Kolmogorov equation. This result is obtained thanks to the introduction of a modified Kolmogorov operator. New a priori estimates on the solutions of the Burgers equation and on the invariant measure are obtained. These are crucial in our argument.
\end{abstract}

\section{Mathematics Subject Classification AMS:}

Key words: stochastic Burgers equation, Kolmogorov equation, m-dissipativity, identité du carré du champ.

\section{Introduction}

We are here concerned with the following stochastic Burgers equation in the interval $[0,1]$ with Dirichlet boundary conditions,

$$
\left\{\begin{aligned}
d X(t, \xi) & =\left(\partial_{x}^{2} X(t, \xi)+\partial_{x}\left(X^{2}(t, \xi)\right)\right) d t+d W(t, \xi), t>0, \xi \in(0,1) \\
X(t, 0) & =X(t, 1)=0, t>0 \\
X(0, \xi) & =x(\xi), \xi \in(0,1) .
\end{aligned}\right.
$$

The unknown $X$ is a real valued process depending on $\xi \in[0,1]$ and $t \geq 0$ and $d W / d t$ is a space-time white noise on $[0,1] \times[0, \infty)$. This equation has been studied by several authors ( see [2], [4], [8], [9], [17], [19]) and it is known that there exists a unique solution with paths in $C\left([0, T] ; L^{2}(0,1)\right)$.

In this article, we want to study (1.1) through the corresponding Kolmogorov equations whose solution is formally given by the transition semigroup associated to (1.1). Numerous articles are devoted to this type of problem ([11], [21], [22], [23]). The aim is to have a better understanding of 
infinite dimensional Kolmogorov operators. In this way, we hope to be able to prove existence and uniqueness results for the transition semigroup in cases when we are not able to solve directly the stochastic equation. In [7] for instance, the existence of a transition semigroup is proved for the three dimensional Navier-Stokes equations by a direct study of the Kolmogorov equation. Other examples of the application of this method are for instance the direct construction of the transition semigroup for the stochastic quantization equations ([18]) or for the porous medium equation $([10])$. Also if the Burgers equation above is perturbed by a term as a nonlinearity of the form $f(X(t, \xi))$ where $f$ is only assumed to be Borelian and bounded, then it is hopeless to solve the equation pathwise but our results below can be used to prove that there exists a unique solution to the Kolmogorov equation. Then, a transition semigroup in $L^{2}(H, \nu)$ can be constructed. Also, one can try to solve the stochastic differential equation (1.1) in the sense of the martingale using use the theory of Dirichlet forms developped in [16], [21] and generalized in [23].

Also, even if we are able to solve directly the stochastic equations, further properties can be derived from the Kolmogorov operator. This is the case for log-Sobolev inequalities. Up to now, we are not able to prove a log-Sobolev inequality for the stochastic Burgers equation. However, we think that the tools developed in this article will be useful for that purpose. Another application is to solve Hamilton-Jacobi equation associated to control problem for the stochastic Burgers equation (1.1).

We rewrite (1.1) as an abstract differential equation in the Hilbert space $H=L^{2}(0,1)$,

$$
\left\{\begin{array}{l}
d X=(A X+b(X)) d t+d W(t) \\
X(0)=x
\end{array}\right.
$$

where

- $A=\partial_{\xi}^{2}, \quad D(A)=H^{2}(0,1) \cap H_{0}^{1}(0,1)$,

- $b(x)=\partial_{\xi}\left(x^{2}\right), \quad D(b)=H_{0}^{1}(0,1)$,

- $W$ is a cylindrical process on $H$, associated to a stochastic basis $\left(\Omega, \mathcal{F}, \mathbb{P},\left(\mathcal{F}_{t}\right)_{t \geq 0}\right)$.

The unique solution to equation (1.2) is denoted by $X(t, x)$. The corresponding transition semigroup $P_{t}$ is then given by,

$$
P_{t} \varphi(x)=\mathbb{E}[\varphi(X(t, x))], \quad t \geq 0, x \in H, \varphi \in B_{b}(H),
$$

where $B_{b}(H)$ is the Banach space of all Borel bounded mappings $\varphi: H \rightarrow \mathbb{R}$ endowed with the sup norm

$$
\|\varphi\|_{0}=\sup _{x \in H}|\varphi(x)|
$$

and $\mathbb{E}$ means expectation.

We know that there exists a unique invariant measure $\nu$ for $P_{t}$, see [8], [9]. The existence follows from Krylov-Boboliubov criterium and an a priori estimates on the solutions. The estimates given 
in section 2.3 can also be used. The uniqueness follows from the Doob theorem. Strong Feller property is a consequence of the results of section 3 - see Remark 3.5 below, irreducibility can be obtained by an approximate controllability argument.

As is well known, $P_{t}$ can be uniquely extended to a contraction Markov semigroup in $L^{2}(H, \nu)$, still denoted by $P_{t}$. We shall denote by $N_{2}$ its infinitesimal generator. The main result of this paper is that $N_{2}$ is the closure in $L^{2}(H, \nu)$ of the Kolmogorov operator $N_{0}$ defined by

$$
N_{0} \varphi(x)=\frac{1}{2} \operatorname{Tr}\left[D^{2} \varphi(x)\right]-(A x+b(x), D \varphi(x)), \quad x \in H, \varphi \in \mathcal{E}_{A}(H),
$$

where $\mathcal{E}_{A}(H)$ (the algebra of all exponential functions) is the linear span of all functions (more precisely the linear span of real and imaginary parts of all functions)

$$
\varphi_{h}(x)=e^{i(h, x)}, \quad x \in H, h \in D(A) .
$$

In other words we show that $N_{2}$ is an extension of $N_{0}$ and that $\mathcal{E}_{A}(H)$ is a core for $N_{2}$. This can be expressed by saying that $N_{0}$ is essentially $m$-dissipative. This result generalizes a previous one, see [6], proved for a coloured noise.

As in [6], the main tool for proving this result is a suitable bound for the differential of the transition semigroup $D P_{t} \varphi(x)$. There, this estimate was a direct consequence of estimates on the derivative $X_{x}(t, x)$ of the process $X(t, x)$ and on exponential moments of $X(t, x)$. In the case of a white noise, we were not able to obtain such bounds. For that purpose, we use here a similar method as in [7] by introducing a Kolmogorov operator with a suitable potential term

$$
\tilde{N} \varphi(x)=N_{0} \varphi(x)-K|x|_{L^{4}}^{4} \varphi(x), \quad x \in H, \varphi \in \mathcal{E}_{A}(H),
$$

where $K$ is sufficiently large, and the corresponding semigroup $S_{t}$ given by the Feynman-Kac formula,

$$
S_{t} \varphi(x)=\mathbb{E}\left(e^{-K \int_{0}^{t}|X(s, x)|_{L^{4}}^{4} d s} \varphi(X(t, x))\right), \quad \varphi \in B_{b}(H), t \geq 0, x \in H .
$$

Thanks to the exponential factor, we are able to find an estimate for $\left|D S_{t} \varphi(x)\right|$ thanks to estimates on the derivative $X_{x}(t, x)$. Moreover, using a generalization of the Bismut-Elworthy formula (see $[3],[13],[15])$, we get smoothing properties for the Feynman-Kac semigroup $\left(S_{t}\right)_{t \geq 0}$. Then, using the identity

$$
P_{t} \varphi=S_{t} \varphi+K \int_{0}^{t} S_{t-s}\left(|\cdot|{ }_{L^{4}}^{4} P_{s} \varphi\right) d s,
$$

we obtain estimates for $D P_{t} \varphi(x)$. In this argument, we face a strong difficulty in the estimation of the moments of the solutions and of the invariant measure. We introduce a new tool to do these estimates. We use a modified Ornstein-Uhlenbeck process as in [5], but the parameter is random here. This is a very powerful tool which, we think, can be used in other situations. It can also be used, for instance, to estimate the Hausdorff dimension of the random attractor of the 
stochastic Burgers equation or of the stochastic Navier-Stokes equations, see Remark 2.4 below. Note that another proof of the finiteness of moments of the solutions is given in [19], it is based on the Hopf-Cole transform and is really specific to the Burgers equation.

We end this section by giving some notations which we will use in what follows. We denote by $|\cdot|$ the norm of $L^{2}(0,1)$. For $p \geq 1,|\cdot|_{L^{p}}$ is the norm of $L^{p}(0,1)$.

The linear operator $A$ is selfadjoint and it possesses a complete orthonormal system $\left\{e_{k}\right\}$ in $H$ of eigenfunctions given by

$$
e_{k}(\xi)=\sqrt{\frac{2}{\pi}} \sin k \xi, \quad \xi \in[0,1], k \in \mathbb{N}
$$

and

$$
A e_{k}=-\pi^{2} k^{2} e_{k}, \quad k \in \mathbb{N} .
$$

For any $\alpha \in \mathbb{R},(-A)^{\alpha}$ is the power of the operator $-A$, which can easily be defined through the eigenbasis above, and $|\cdot|_{\alpha}$ is the norm of $D\left((-A)^{\alpha / 2}\right)$ which is equivalent to the norm of $H^{\alpha}(0,1)$. We have $|\cdot|_{0}=|\cdot|=|\cdot|_{L^{2}}$.

The cylindrical Wiener process $W(t), t \in \mathbb{R}$ in $H$ can be formally written as

$$
W(t)=\sum_{k=1}^{\infty} \beta_{k}(t) e_{k}, \quad t \geq 0,
$$

where $\left\{\beta_{k}\right\}$ is a sequence of mutually independent standard Brownian motions in some probability space $(\Omega, \mathcal{F}, \mathbb{P})$.

We shall often use the classical interpolatory estimate,

$$
|x|_{\beta} \leq|x|_{\alpha}^{\frac{\gamma-\beta}{\gamma-\alpha}}|x|_{\gamma}^{\frac{\beta-\alpha}{\gamma-\alpha}}, \quad \alpha<\beta<\gamma
$$

and the Agmon estimate

$$
|x|_{L^{\infty}} \leq|x|_{L^{2}}^{\frac{1}{2}}|x|_{H^{1}}^{\frac{1}{2}}
$$

Finally, we recall that the semigroup $e^{t A}$ acts on $L^{p}(0,1)$ for all $p \geq 1$ and that the following estimate holds (by the Poincaré inequality),

$$
\left|e^{t A} x\right|_{L^{p}} \leq e^{-\lambda_{p} t}|x|_{L^{p}}, \quad t \geq 0, x \in L^{p},
$$

where

$$
\lambda_{p}=\frac{2(p-1)}{p} \pi^{2}, \quad p \in(1, \infty) .
$$




\section{Estimate of moments of $X(t, x)$}

\subsection{Estimate of a modified Ornstein-Uhlenbeck process}

Here we introduce a modified Ornstein-Uhlenbeck process replacing $A$ with $A-\alpha$ with $\alpha \geq 0$. We set, for any $\alpha \geq 0$,

$$
z_{\alpha}(t)=\int_{0}^{t} e^{(t-s)(A-\alpha)} d W(s), \quad t \geq 0
$$

it is the mild solution of the equation

$$
d z_{\alpha}(t)=(A-\alpha) z_{\alpha}(t) d t+d W(t), \quad z_{\alpha}(0)=0 .
$$

This type of modified Ornstein-Uhlenbeck process was introduced in [5] in order to study the existence of random attractors for the stochastic Navier-Stokes equation. In this present work, we use it in a different way and need more precise estimates on the dependance of $z_{\alpha}$ with respect to $\alpha$. We use the following result.

Proposition 2.1 Let $p \geq 1, \varepsilon \in(0,1 / 4), \delta \in(0,1)$. Then there exists a random variable $K_{\varepsilon, \delta, p}$ such that for all $\alpha>0$ and $t \geq 0$ we have

$$
\left|z_{\alpha}(t)\right|_{L^{p}} \leq \alpha^{-\frac{1}{4}+\varepsilon}\left(1+t^{\delta}\right) K_{\varepsilon, \delta, p},
$$

and

$$
\mathbb{E}\left(K_{\varepsilon, \delta, p}^{k}\right)<+\infty, \quad \text { for all } k \geq 1 \text {. }
$$

Proof. Using the identity

$$
e^{-(t-s) \alpha}=1-\alpha \int_{s}^{t} e^{-(t-\tau) \alpha} d \tau
$$

we can write

$$
\begin{aligned}
z_{\alpha}(t) & =\int_{0}^{t} e^{(t-s) A} d W(s)-\alpha \int_{0}^{t} e^{(t-s) A} \int_{s}^{t} e^{-(t-\tau) \alpha} d \tau d W(s) \\
& =\int_{0}^{t} e^{(t-s) A} d W(s)-\alpha \int_{0}^{t} e^{(t-\tau)(A-\alpha)} \int_{0}^{\tau} e^{(\tau-s) A} d W(s) d \tau .
\end{aligned}
$$

We now use the factorization method (see [11], section 5.3) writing for $\beta \in(0,1)$,

$$
\int_{0}^{t} e^{(t-s) A} d W(s)=\frac{\sin \pi \beta}{\pi} \int_{0}^{t}(t-\sigma)^{\beta-1} e^{(t-\sigma) A} Y(\sigma) d \sigma
$$

where

$$
Y(\sigma)=\int_{0}^{\sigma}(\sigma-s)^{-\beta} e^{(\sigma-s) A} d W(s)
$$


It follows that

$$
\begin{aligned}
z_{\alpha}(t)= & \frac{\sin \pi \beta}{\pi}\left[\int_{0}^{t}(t-\sigma)^{\beta-1} e^{(t-\sigma) A} Y(\sigma) d \sigma\right. \\
& \left.-\alpha \int_{0}^{t} e^{(t-\tau)(A-\alpha)} \int_{0}^{\tau}(\tau-\sigma)^{\beta-1} e^{(\tau-\sigma) A} Y(\sigma) d \sigma d \tau\right] \\
= & \frac{\sin \pi \beta}{\pi} \int_{0}^{t} e^{(t-\sigma) A} Y(\sigma)\left[(t-\sigma)^{\beta-1}-\alpha \int_{\sigma}^{t}(\tau-\sigma)^{\beta-1} e^{-\alpha(t-\tau)} d \tau\right] d \sigma .
\end{aligned}
$$

We are going to estimate the factor inside brackets

$$
L:=(t-\sigma)^{\beta-1}-\alpha \int_{\sigma}^{t}(\tau-\sigma)^{\beta-1} e^{-\alpha(t-\tau)} d \tau .
$$

We have, using the change of variable $\tau-\sigma=(t-\sigma) \rho$,

$$
\begin{aligned}
& L=\alpha \int_{\sigma}^{t}\left((t-\sigma)^{\beta-1}-(\tau-\sigma)^{\beta-1}\right) e^{-\alpha(t-\tau)} d \tau+(t-\sigma)^{\beta-1} e^{-\alpha(t-\sigma)} \\
& =\alpha(t-\sigma)^{\beta} \int_{0}^{1}\left(1-\rho^{\beta-1}\right) e^{-\alpha(1-\rho)(t-\sigma)} d \rho+(t-\sigma)^{\beta-1} e^{-\alpha(t-\sigma)} .
\end{aligned}
$$

For any $s \geq 0$ we can write

$$
\begin{aligned}
\int_{0}^{1}\left(1-\rho^{\beta-1}\right) e^{-(1-\rho) s} d \rho & \leq e^{-s / 2} \int_{0}^{1 / 2}\left(1-\rho^{\beta-1}\right) d \rho+c(\beta) \int_{1 / 2}^{1}(1-\rho) e^{-(1-\rho) s} d \rho \\
& \leq c(\beta) s^{-2} .
\end{aligned}
$$

We deduce that the following estimate holds for $\gamma \in(0,1)$

$$
|L| \leq c(\gamma, \beta) \alpha^{-\gamma}(t-\sigma)^{\beta-1-\gamma}+(t-\sigma)^{\beta-1} e^{-\alpha(t-\sigma)} .
$$

It follows, by (1.10), that for all $p \geq 1$

$$
\begin{aligned}
& \left|z_{\alpha}(t)\right|_{L^{p}} \\
& \leq c(\gamma, \beta) \int_{0}^{t}\left|e^{(t-\sigma) A} Y(\sigma)\right|_{L^{p}}\left(\alpha^{-\gamma}(t-\sigma)^{\beta-1-\gamma}+(t-\sigma)^{\beta-1} e^{-\alpha(t-\sigma)}\right) d \sigma \\
& \leq c(\gamma, \beta) \int_{0}^{t} e^{-\lambda_{p}(t-\sigma)}|Y(\sigma)|_{L^{p}}\left[\alpha^{-\gamma}(t-\sigma)^{\beta-1-\gamma}+(t-\sigma)^{\beta-1} e^{-\alpha(t-\sigma)}\right] d \sigma .
\end{aligned}
$$


Consequently, thanks to the Hölder inequality we obtain

$$
\begin{aligned}
\left|z_{\alpha}(t)\right|_{L^{p}} \leq & c(\gamma, \beta)\left(\int_{0}^{t} e^{-\lambda_{p}(t-\sigma)}|Y(\sigma)|_{L^{p}}^{2 m} d \sigma\right)^{\frac{1}{2 m}} \\
& \times\left[\alpha^{-\gamma}\left(\int_{0}^{t} e^{-\lambda_{p}(t-\sigma)}(t-\sigma)^{\frac{2 m}{2 m-1}(\beta-1-\gamma)} d \sigma\right)^{\frac{2 m-1}{2 m}}\right. \\
& \left.+\left(\int_{0}^{t} e^{-\lambda_{p}(t-\sigma)}(t-\sigma)^{\frac{2 m}{2 m-1}(\beta-1)} e^{-(t-\sigma) \frac{2 m \alpha}{2 m-1}} d \sigma\right)^{\frac{2 m-1}{2 m}}\right] .
\end{aligned}
$$

Note that

$$
\int_{0}^{t} e^{-\lambda_{p}(t-\sigma)}(t-\sigma)^{\frac{2 m}{2 m-1}(\beta-1-\gamma)} d \sigma \leq \int_{0}^{+\infty} e^{-\lambda_{p} u} u^{\frac{2 m}{2 m-1}(\beta-1-\gamma)} d u<+\infty
$$

provided $\frac{2 m}{2 m-1}(\beta-1-\gamma)>-1$, that is

$$
\beta>\gamma+\frac{1}{2 m}
$$

Moreover

$$
\begin{aligned}
& \int_{0}^{t} e^{-\lambda_{p}(t-\sigma)}(t-\sigma)^{\frac{2 m}{2 m-1}(\beta-1)} e^{-(t-\sigma) \frac{2 m \alpha}{2 m-1}} d \sigma \leq \int_{0}^{+\infty} u^{\frac{2 m}{2 m-1}(\beta-1)} e^{-u \frac{2 m \alpha}{2 m-1}} d u \\
& =\alpha^{-1-\frac{2 m}{2 m-1}(\beta-1)} \int_{0}^{+\infty} u^{\frac{2 m}{2 m-1}(\beta-1)} e^{-\frac{2 m u}{2 m-1}} d u \leq c \alpha^{\frac{1-2 \beta m}{2 m-1}},
\end{aligned}
$$

provided $\frac{2 m}{2 m-1}(\beta-1)>-1$, that is

$$
\beta>\frac{1}{2 m} .
$$

In conclusion, choosing $\beta \in(1 / 2 m, 1), 0<\gamma<\beta-1 / 2 m$, we have proved that

$$
\left|z_{\alpha}(t)\right|_{L^{p}} \leq c(\gamma, \beta, m)\left(\alpha^{-\gamma}+\alpha^{-\beta+\frac{1}{2 m}}\right)\left(\int_{0}^{t} e^{-\lambda_{p}(t-\sigma)}|Y(\sigma)|_{L^{p}}^{2 m} d \sigma\right)^{\frac{1}{2 m}}
$$

Let us now estimate the moments of $Y$. We have

$$
Y(\sigma, \xi)=\sum_{k=1}^{\infty} \int_{0}^{\sigma}(\sigma-s)^{-\beta} e^{-\pi^{2} k^{2}(\sigma-s)} e_{k}(\xi) d \beta_{k}(s), \quad \sigma \in \mathbb{R}, \xi \in[0,1]
$$


so that

$$
\mathbb{E}\left(|Y(\sigma, \xi)|^{2}\right)=\sum_{k=1}^{\infty} \int_{0}^{\sigma} s^{-2 \beta} e^{-2 \pi^{2} k^{2} s} e_{k}^{2}(\xi) d s \leq c \sum_{k=1}^{\infty} \frac{1}{k^{2-4 \beta}}<+\infty,
$$

provided $\beta<\frac{1}{4}$. By Gaussianity of $Y$, it follows that

$$
\mathbb{E}\left(|Y(\sigma)|_{L^{p}}^{p}\right) \leq c_{p}\left(\sum_{k=1}^{\infty} \int_{0}^{\sigma} e^{-2 \pi^{2} k^{2} s} s^{-2 \beta} d s\right)^{\frac{p}{2}} \leq c_{p}\left(\sum_{k=1}^{\infty} k^{-2+4 \beta}\right)^{\frac{p}{2}},
$$

where $c_{p}$ is a finite constant

Using once again the fact that $Y$ is Gaussian, yields

$$
\mathbb{E}\left(|Y(\sigma)|_{L^{p}}^{k}\right) \leq c(p, k)
$$

for all $p, k \geq 1$.

Now, by the Hölder inequality it follows that

$$
\begin{aligned}
& \int_{0}^{t} e^{-\lambda(t-\sigma)}|Y(\sigma)|_{L^{p}}^{2 m} d \sigma \\
& \leq\left(\int_{0}^{+\infty}\left(1+\sigma^{2}\right)^{-1}|Y(\sigma)|_{L^{p}}^{4 m} d \sigma\right)^{\frac{1}{2}}\left(\int_{0}^{t} e^{-2 \lambda(t-\sigma)}\left(1+\sigma^{2}\right) d \sigma\right)^{\frac{1}{2}} \\
& \leq c(1+t)\left(\int_{0}^{+\infty}\left(1+\sigma^{2}\right)^{-1}|Y(\sigma)|_{L^{p}}^{4 m} d \sigma\right)^{\frac{1}{2}} .
\end{aligned}
$$

Finally by (2.5), we obtain

$$
\left|z_{\alpha}(t)\right|_{L^{p}} \leq c(\gamma, \beta, m)\left(\alpha^{-\gamma}+\alpha^{-\beta+\frac{1}{2 m}}\right)(1+t)^{\frac{1}{2 m}}\left(\int_{0}^{+\infty}\left(1+\sigma^{2}\right)^{-1}|Y(\sigma)|_{L^{p}}^{4 m} d \sigma\right)^{\frac{1}{4 m}} .
$$

To prove the result we choose $\gamma, \beta, m$ such that

$$
\gamma \in(0,1), \quad \frac{1}{2 m}<\min \{\delta, \varepsilon / 2\}, \quad \frac{1}{4}-\varepsilon / 2<\gamma+\varepsilon / 2<\beta<\frac{1}{4} .
$$

Notice that, with this choice, (2.3), (2.4) hold and we have

$$
\left|z_{\alpha}(t)\right|_{L^{p}} \leq \alpha^{-\frac{1}{4}+\varepsilon}\left(1+t^{\delta}\right) K_{\varepsilon, \delta, p},
$$

with

$$
K_{\varepsilon, \delta, p}=c(\varepsilon, \delta)\left(\int_{0}^{+\infty}\left(1+\sigma^{2}\right)^{-1}|Y(\sigma)|_{L^{p}}^{4 m} d \sigma\right)^{\frac{1}{4 m}}, \quad t \geq 0
$$

In view of $(2.6)$, we have

$$
\mathbb{E}\left(K_{\varepsilon, \delta, p}^{k}\right)<\infty
$$

for all $k$. The proof is complete. 


\subsection{Estimate of the solution $X(t, x)$}

Proposition 2.2 For all $p, k \geq 1$ there exist $c_{1}(p, k), c_{2}(p, k)$ such that for all $x \in L^{p}(0,1)$ and all $T \geq 0$ we have

$$
\mathbb{E}\left(\sup _{t \in[0, T]}|X(t, x)|_{L^{p}}^{k}\right) \leq c_{1}(p, k) e^{c_{2}(p, k) T}\left(1+|x|_{L^{p}}^{k}\right) .
$$

Proof. We set $Y(t)=X(t, x)-z_{\alpha}(t), t \geq 0$, so that $Y$ fulfills

$$
\frac{d}{d t} Y(t)=A Y(t)+b\left(Y(t)+z_{\alpha}(t)\right)+\alpha z_{\alpha}(t)
$$

Multiplying both sides by $p|Y(t)|^{p-2} Y(t)$, integrating in $[0,1]$ and then integrating by parts, yields

$$
\begin{aligned}
& \frac{d}{d t}|Y(t)|_{L^{p}}^{p}+p(p-1) \int_{0}^{1}|Y(t)|^{p-2}\left|\partial_{\xi} Y(t)\right|^{2} d \xi \\
& =p \int_{0}^{1}|Y(t)|^{p-2} Y(t) \partial_{\xi}\left[\left(Y(t)+z_{\alpha}(t)\right)^{2}\right] d \xi+p \alpha \int_{0}^{1}|Y(t)|^{p-2} Y(t) z_{\alpha}(t) d \xi \\
& =-p(p-1) \int_{0}^{1}|Y(t)|^{p-2} \partial_{\xi} Y(t)\left(Y^{2}(t)+2 Y(t) z_{\alpha}(t)+z_{\alpha}^{2}(t)\right) d \xi \\
& +p \alpha \int_{0}^{1}|Y(t)|^{p-2} Y(t) z_{\alpha}(t) d \xi \\
& =-2 p(p-1) \int_{0}^{1}|Y(t)|^{p-2} Y(t) \partial_{\xi} Y(t) z_{\alpha}(t) d \xi \\
& -p(p-1) \int_{0}^{1}|Y(t)|^{p-2} \partial_{\xi} Y(t) z_{\alpha}^{2}(t) d \xi+p \alpha \int_{0}^{1}|Y(t)|^{p-2} Y(t) z_{\alpha}(t) d \xi \\
& :=I_{1}+I_{2}+I_{3}
\end{aligned}
$$

since $\int_{0}^{1}|Y(t)|^{p} Y(t) \partial_{\xi} Y(t) d \xi=0$.

Let us estimate $I_{1}$. By Hölder inequality we have

$$
\int_{0}^{1}|Y(t)|^{p-2} Y(t) \partial_{\xi} Y(t) z_{\alpha}(t) d \xi \leq\left.\left.\left.\left.|| Y(t)\right|^{\frac{p}{2}}\right|_{L^{4}}|| Y(t)\right|^{\frac{p}{2}-1} \partial_{\xi} Y(t)\right|_{L^{2}}\left|z_{\alpha}(t)\right|_{L^{4}}
$$

We then use Sobolev embedding theorem and the interpolatory inequality (1.8) to obtain

$$
\left.\left.|| Y(t)\right|^{\frac{p}{2}}\right|_{L^{4}} \leq\left.\left. c|| Y(t)\right|^{\frac{p}{2}}\right|_{H^{\frac{1}{4}}} \leq\left.\left.\left.\left. c|| Y(t)\right|^{\frac{p}{2}}\right|_{L^{2}} ^{\frac{3}{4}}|| Y(t)\right|^{\frac{p}{2}}\right|_{H^{1}} ^{\frac{1}{4}} \text {. }
$$


Moreover $\left.\left.|| Y(t)\right|^{\frac{p}{2}}\right|_{L^{2}}=|Y(t)|_{L^{p}}^{\frac{p}{2}}$ and, thanks to Poincaré inequality,

$$
\left.\left.|| Y(t)\right|^{\frac{p}{2}}\right|_{H^{1}} \leq\left.\left. c\left|\partial_{\xi}\right| Y(t)\right|^{\frac{p}{2}}\right|_{L^{2}}=\left.\left.c|| Y(t)\right|^{\frac{p}{2}-1} \partial_{\xi} Y(t)\right|_{L^{2}} .
$$

It follows

$$
|I|_{1} \leq\left.\left. c|Y(t)|_{L^{p}}^{\frac{3 p}{8}}|| Y(t)\right|^{\frac{p}{2}-1} \partial_{\xi} Y(t)\right|_{L^{2}} ^{\frac{5}{4}}\left|z_{\alpha}\right|_{L^{4}}
$$

Now, using the estimate

$$
a b \leq \frac{3}{8} a^{\frac{8}{3}}+\frac{5}{8} b^{\frac{8}{5}}, \quad a, b \geq 0
$$

we find that

$$
\left|I_{1}\right| \leq\left.\left.\frac{p(p-1)}{4}|| Y(t)\right|^{\frac{p}{2}-1} \partial_{\xi} Y(t)\right|_{L^{2}} ^{2}+c\left|z_{\alpha}\right|_{L^{4}}^{\frac{8}{3}}|Y(t)|_{L^{p}}^{p}
$$

Concerning $I_{2}$ we have, by Hölder inequality,

$$
\left|I_{2}\right| \leq\left.\left. c|| Y(t)\right|^{\frac{p}{2}-1} \partial_{\xi} Y(t)\right|_{L^{2}}\left|z_{\alpha}\right|_{L^{4}}^{2}\left|Y(t)^{\frac{p}{2}}\right|_{L^{\infty}}
$$

But, by (1.9),

$$
\left|Y(t)^{\frac{p}{2}}\right|_{L^{\infty}} \leq\left|Y(t)^{\frac{p}{2}}\right|_{L^{2}}^{1 / 2}\left|Y(t)^{\frac{p}{2}}\right|_{H^{1}}^{1 / 2} \leq\left.\left. c|Y(t)|_{L^{p}}^{\frac{p}{4}}|| Y(t)\right|^{\frac{p}{2}-1} \partial_{\xi} Y(t)\right|_{L^{2}} ^{\frac{1}{2}}
$$

Consequently

$$
\left|I_{2}\right| \leq\left.\left. c|Y(t)|_{L^{p}}^{\frac{p}{4}}|| Y(t)\right|^{\frac{p}{2}-1} \partial_{\xi} Y(t)\right|_{L^{2}} ^{\frac{3}{2}}\left|z_{\alpha}(t)\right|_{L^{4}}^{2}
$$

Now, using the estimate

$$
a b \leq \frac{3}{4} a^{\frac{4}{3}}+\frac{1}{4} b^{4}, \quad a, b \geq 0
$$

we find that

$$
\left|I_{2}\right| \leq\left.\left.\frac{p(p-1)}{4}|| Y(t)\right|^{\frac{p}{2}-1} \partial_{\xi} Y(t)\right|_{L^{2}} ^{2}+c\left|z_{\alpha}(t)\right|_{L^{4}}^{8}|Y(t)|_{L^{p}}^{p} .
$$

Finally, for $I_{3}$ we have

$$
\left|I_{3}\right| \leq \alpha|Y(t)|_{L^{p}}^{p-1}\left|z_{\alpha}(t)\right|_{L^{p}} \leq\left|z_{\alpha}(t)\right|_{L^{p}}\left(|Y(t)|_{L^{p}}^{p}+c \alpha^{p}\right) .
$$

By (2.8), (2.9) and (2.10) it follows that

$$
\begin{aligned}
& \frac{d}{d t}|Y(t)|_{L^{p}}^{p}+\frac{1}{2} p(p-1) \int_{0}^{1}|Y(t)|^{p-2}\left|\partial_{\xi} Y(t)\right|^{2} d \xi \\
& \leq c\left(\left|z_{\alpha}(t)\right|_{L^{4}}^{\frac{8}{3}}+\left|z_{\alpha}(t)\right|_{L^{4}}^{8}+\left|z_{\alpha}(t)\right|_{L^{p}}\right)|Y(t)|_{L^{p}}^{p}+c \alpha^{p}\left|z_{\alpha}(t)\right|_{L^{p}}^{p} .
\end{aligned}
$$


We assume from now on for simplicity that $p \geq 4$. Then we have

$$
\frac{d}{d t}|Y(t)|_{L^{p}}^{p} \leq\left(\left|z_{\alpha}(t)\right|_{L^{p}}^{8}+1\right)|Y(t)|_{L^{p}}^{p}+c \alpha^{p}\left|z_{\alpha}(t)\right|_{L^{p}}
$$

Now, in view of Proposition 2.1, we can choose $\alpha$ such that

$$
\sup _{t \in[0, T]}\left|z_{\alpha}(t)\right|_{L^{p}}^{8} \leq 1
$$

For instance we can take

$$
\alpha=\left((1+T)^{1 / 2} K_{\frac{1}{8}, \frac{1}{2}, p}\right)^{8}+1
$$

Consequently,

$$
\frac{d}{d t}|Y(t)|_{L^{p}}^{p} \leq 2 c|Y(t)|_{L^{p}}^{p}+c \alpha^{p}
$$

and

$$
|Y(t)|_{L^{p}}^{p} \leq e^{2 c t}\left(|x|_{L^{p}}^{p}+c \alpha^{p}\right)
$$

Therefore, we have

$$
\sup _{t \in[0, T]}|Y(t)|_{L^{p}}^{p} \leq e^{2 c T}\left(|x|_{L^{p}}^{p}+c(1+T)^{4 p} K_{\frac{1}{8}, \frac{1}{2}, p}^{8 p}\right)
$$

Since

$$
\sup _{t \in[0, T]}\left|z_{\alpha}(t)\right|_{L^{p}} \leq \alpha^{-\frac{1}{8}}(1+T) K_{\frac{1}{8}, \frac{1}{2}, p}
$$

we have

$$
\sup _{t \in[0, T]}|X(t, x)|_{L^{p}}^{p} \leq e^{c(p) T}\left(|x|_{L^{p}}+K_{p}\right)
$$

where $K_{p}$ is a random variable having all moment finite. The result follows.

\subsection{Estimate of moments of $\nu$}

We recall that $\nu$ represents the invariant measure of $X(t, x)$.

Proposition 2.3 For all $p, k \geq 1$ we have

$$
\int_{H}|x|_{L^{p}}^{k} \nu(d x)<+\infty
$$

Proof. We first notice that, by the Poincaré inequality, we have

$$
\left(\frac{p}{2}\right)^{2} \int_{0}^{1}|y|^{p-2}\left|\partial_{\xi} y\right|^{2} d \xi=\left.\left.\int_{0}^{1}\left|\partial_{\xi}\right| y\right|^{\frac{p}{2}}\right|^{2} d \xi \geq \pi^{2}|y|_{L^{p}}^{p} .
$$


Consequently by (2.11)

$$
\frac{d}{d t}|Y(t)|_{L^{p}}^{p}+\frac{2(p-1) \pi^{2}}{p}|Y(t)|_{L^{p}}^{p} \leq c\left(\left|z_{\alpha}(t)\right|_{L^{4}}^{\frac{8}{3}}+\left|z_{\alpha}(t)\right|_{L^{4}}^{8}+\left|z_{\alpha}(t)\right|_{L^{p}}\right)|Y(t)|_{L^{p}}^{p}+c \alpha^{p}\left|z_{\alpha}(t)\right|_{L^{p}} .
$$

We assume for simplicity that $p \geq 4$ so that

$$
c\left(\left|z_{\alpha}(t)\right|_{L^{4}}^{\frac{8}{3}}+\left|z_{\alpha}(t)\right|_{L^{p}}^{8}+\left|z_{\alpha}(t)\right|_{L^{4}}\right) \leq c\left|z_{\alpha}(t)\right|_{L^{p}}^{8}+\frac{(p-1) \pi^{2}}{2 p} .
$$

We choose

$$
\alpha=\left(\frac{2 p c}{(p-1) \pi^{2}} K_{\frac{1}{8}, 1, p}\right)^{8}+1
$$

then for all $t \in[0,1]$

$$
c\left(\left|z_{\alpha}(t)\right|_{L^{4}}^{\frac{8}{3}}+\left|z_{\alpha}(t)\right|_{L^{4}}^{8}+\left|z_{\alpha}(t)\right|_{L^{p}}\right) \leq \frac{(p-1) \pi^{2}}{p} .
$$

Therefore

$$
\frac{d}{d t}|Y(t)|_{L^{p}}^{p}+\frac{(p-1) \pi^{2}}{p}|Y(t)|_{L^{p}}^{p} \leq c \alpha^{p}=\overline{K_{1}}(\omega)
$$

where $\overline{K_{1}}$ is a random variable having all moments finite. We deduce that

$$
|Y(1)|_{L^{p}}^{p} \leq e^{-\frac{(p-1) \pi^{2}}{p}}\left(|x|_{L^{p}}^{p}+\overline{K_{1}}(\omega)\right) .
$$

We use the following inequality for $\varepsilon>0, k \geq 1$,

$$
|a+b|^{k} \leq(1+\varepsilon) a^{k}+c(\varepsilon, k) b^{k}, \quad a, b \in \mathbb{R} .
$$

We get

$$
\begin{aligned}
|X(1, x)|_{L^{p}}^{p} & \leq e^{-\frac{(p-1) \pi^{2}}{2 p}}|Y(1)|_{L^{p}}^{p}+c(p)\left|z_{\alpha}(1)\right|_{L^{p}}^{p} \\
& \leq e^{-\frac{(p-1) \pi^{2}}{2 p}}\left(|x|_{L^{p}}^{p}+\overline{K_{1}}(\omega)\right)+c(p) K_{\frac{1}{8}, 1, p}
\end{aligned}
$$

(we have $\alpha \geq 1$ ). Using again (2.13) we find that

$$
\left.|X(1, x)|_{L^{p}}^{k} \leq e^{-\frac{(p-1) \pi^{2}}{4 p}}|x|_{L^{p}}^{k}+\overline{K_{2}}(\omega)\right)
$$

where $\overline{K_{2}}$ is a random variable having all moments finite. Integrating with respect to $\omega$, then with respect to $\nu$ and using invariance of $\nu$ yields

$$
\int_{H}|x|_{L^{p}}^{k} \nu(d x) \leq \frac{1}{1-e^{-\frac{(p-1) \pi^{2}}{4 p}}} \mathbb{E}\left(\overline{K_{2}}\right) .
$$


Remark 2.4 Using the results of [5], it is not difficult to show that the stochastic Burgers equation considered here posseses a random attractor. The proof is similar to the proof given in this article for the stochastic Navier-Stokes equations. An unsolved problem is to prove that this random attractor has finite Hausdorff dimension. In fact using the result of [14] and the techniques used in the proof of Proposition 2.3, we can apply the result of [14] and prove that the Hausdorff dimension of the random attractor of the stochastic Burgers equation has finite Hausdorff dimension. Moreover, this dimension can be majorized in terms of the global Lyapunov exponents. The same argument can be used for the stochastic Navier-Stokes equations.

\section{Estimate of $D P_{t} \varphi(x)$}

We shall proceed in three steps to bound $D P_{t} \varphi(x)$. In section 3.1 we estimate $\eta^{h}(t, x)$, the derivative of $X(t, x)$ in the direction $h \in H$. In section 3.2, we use this estimate to bound $D S_{t} \varphi(x)$, where the semigroup $S_{t}$ is defined in (1.6). Finally in section 3.3, we obtain the required estimate for $D P_{t} \varphi(x)$ using the identity (1.7).

We shall present only formal proofs. They could be justified using a suitable approximation of the Burgers equation - for instance by Galerkin approximations as was done in [7].

\subsection{Estimate of $\eta^{h}(t, x)$}

Let us first notice that $\eta^{h}(t, x)$ fulfills the equation

$$
\left\{\begin{array}{l}
\frac{d}{d t} \eta^{h}(t, x)=A \eta^{h}(t, x)+b^{\prime}(X(t, x)) \eta^{h}(t, x), \\
\eta^{h}(0), x=h,
\end{array}\right.
$$

where

$$
b^{\prime}(X(t, x)) \eta^{h}(t, x)=2 \partial_{\xi}\left(X(t, x) \eta^{h}(t, x)\right) .
$$

Proposition 3.1 For any $\alpha \in[-1,0]$ there exists $c=c(\alpha)>0$ such that

$$
e^{-c \int_{0}^{t}|X(s, x)|_{L^{4}}^{\frac{8}{3}} d s}\left|\eta^{h}(t, x)\right|_{\alpha}^{2}+\int_{0}^{t} e^{-c \int_{s}^{t}|X(\tau, x)|_{L^{4}}^{\frac{8}{3}} d \tau}\left|\eta^{h}(s, x)\right|_{1+\alpha}^{2} d s \leq|h|_{\alpha}^{2},
$$

for all $t \geq 0, x, h \in H$.

Proof. We divide the proof in two parts.

Case 1. $\alpha \in[-3 / 4,0]$. 
Multiplying both sides of the first identity in $(3.1)$ by $(-A)^{\alpha} \eta^{h}(t, x)$, integrating in $[0,1]$, then integrating by parts and using the Hölder inequality, yields

$$
\begin{aligned}
\frac{1}{2} \frac{d}{d t}\left|\eta^{h}(t, x)\right|_{\alpha}^{2}+\left|\eta^{h}(t, x)\right|_{1+\alpha}^{2} & =2 \int_{0}^{1} \partial_{\xi}\left(X(t, x) \eta^{h}(t, x)\right)(-A)^{\alpha} \eta^{h}(t, x) d \xi \\
& =-2 \int_{0}^{1} X(t, x) \eta^{h}(t, x) \partial_{\xi}\left[(-A)^{\alpha} \eta^{h}(t, x)\right] d \xi \\
& \leq 2|X(t, x)|_{L^{4}}\left|\eta^{h}(t, x)\right|_{L^{4}}\left|\eta^{h}(t, x)\right|_{1+2 \alpha} .
\end{aligned}
$$

Since $H^{\frac{1}{4}}(0,1) \subset L^{4}(0,1)$, we have, using the interpolatory estimate (1.8)

$$
\left|\eta^{h}(t, x)\right|_{L^{4}} \leq c\left|\eta^{h}(t, x)\right|_{\frac{1}{4}} \leq c\left|\eta^{h}(t, x)\right|_{\alpha}^{\frac{3}{4}+\alpha}\left|\eta^{h}(t, x)\right|_{1+\alpha}^{\frac{1}{4}-\alpha}
$$

and

$$
\left|\eta^{h}(t, x)\right|_{1+2 \alpha} \leq c\left|\eta^{h}(t, x)\right|_{\alpha}^{-\alpha}\left|\eta^{h}(t, x)\right|_{1+\alpha}^{1+\alpha}
$$

since $\alpha \in[-3 / 4,0]$. Therefore, it results

$$
\begin{aligned}
\frac{d}{d t}\left|\eta^{h}(t, x)\right|_{\alpha}^{2}+\left|\eta^{h}(t, x)\right|_{1+\alpha}^{2} & \leq c|X(t, x)|_{L^{4}}\left|\eta^{h}(t, x)\right|_{\alpha}^{\frac{3}{4}}\left|\eta^{h}(t, x)\right|_{1+\alpha}^{\frac{5}{4}} \\
& \leq c|X(t, x)|_{L^{4}}^{\frac{8}{3}}\left|\eta^{h}(t, x)\right|_{\alpha}^{2}+\frac{1}{2}\left|\eta^{h}(t, x)\right|_{1+\alpha}^{2}
\end{aligned}
$$

where we have used (2.6). Therefore, (3.2) follows by integration in time.

Case 2. $\alpha \in[-1,-1 / 4]$.

Using once again the embedding $H^{\frac{1}{4}}(0,1) \subset L^{4}(0,1)$, we have that

$$
\begin{aligned}
\frac{1}{2} \frac{d}{d t}\left|\eta^{h}(t, x)\right|_{\alpha}^{2}+\left|\eta^{h}(t, x)\right|_{1+\alpha}^{2} & =-2 \int_{0}^{1} X(t, x) \eta^{h}(t, x) \partial_{\xi}\left[(-A)^{\alpha} \eta^{h}(t, x)\right] d \xi \\
& \leq 2|X(t, x)|_{L^{4}}\left|\eta^{h}(t, x)\right|_{L^{2}}\left|\partial_{\xi}(-A)^{\alpha} \eta^{h}(t, x)\right|_{L^{4}} \\
& \leq c|X(t, x)|_{L^{4}}\left|\eta^{h}(t, x)\right|_{L^{2}}\left|\partial_{\xi}(-A)^{\alpha} \eta^{h}(t, x)\right|_{\frac{1}{4}} \\
& \leq c|X(t, x)|_{L^{4}}\left|\eta^{h}(t, x)\right|_{L^{2}}\left|\eta^{h}(t, x)\right|_{\frac{5}{4}+2 \alpha} .
\end{aligned}
$$

Now the interpolatory estimate (1.8) yields

$$
\left|\eta^{h}(t, x)\right|_{L^{2}} \leq\left|\eta^{h}(t, x)\right|_{\alpha}^{1+\alpha}\left|\eta^{h}(t, x)\right|_{1+\alpha}^{-\alpha}
$$


and

$$
\left|\eta^{h}(t, x)\right|_{\frac{5}{4}+2 \alpha} \leq\left|\eta^{h}(t, x)\right|_{\alpha}^{-\alpha-\frac{1}{4}}\left|\eta^{h}(t, x)\right|_{1+\alpha}^{\frac{5}{4}+\alpha},
$$

since $\alpha \leq-\frac{1}{4}$. It follows that, using once again (2.7),

$$
\begin{aligned}
\frac{d}{d t}\left|\eta^{h}(t, x)\right|_{\alpha}^{2}+\left|\eta^{h}(t, x)\right|_{1+\alpha}^{2} & \leq c|X(t, x)|_{L^{4}}\left|\eta^{h}(t, x)\right|_{\alpha}^{\frac{3}{4}}\left|\eta^{h}(t, x)\right|_{1+\alpha}^{\frac{5}{4}} \\
& \leq c|X(t, x)|_{L^{4}}^{\frac{8}{3}}\left|\eta^{h}(t, x)\right|_{\alpha}^{2}+\frac{1}{2}\left|\eta^{h}(t, x)\right|_{1+\alpha}^{2}
\end{aligned}
$$

and (3.2) follows.

\subsection{Estimate of $D S_{t} \varphi(x)$}

We shall use the following notation

$$
\|\varphi\|_{0, L^{4}, k}:=\sup _{x \in L^{4}} \frac{|\varphi(x)|}{1+|x|_{L^{4}}^{k}} .
$$

We know from [13] that for any $\varphi \in C_{b}(H), S_{t}^{m} \varphi$ is differentiable in any direction $h \in H$ and its derivative $D S_{t} \varphi(x) \cdot h$ is given by

$$
\begin{aligned}
& D S_{t} \varphi(x) \cdot h \\
& =\frac{1}{t} \mathbb{E}\left(e^{-K \int_{0}^{t}|X(s, x)|_{L^{4}}^{4} d s} \varphi(X(t, x)) \int_{0}^{t}\left(\eta^{h}(s, x), d W(s)\right)\right) \\
& -4 K \mathbb{E}\left(e^{-K \int_{0}^{t}|X(s, x)|_{L^{4}}^{4} d s} \varphi(X(t, x)) \int_{0}^{t}\left(1-\frac{s}{t}\right)\left(X^{3}(s, x), \eta^{h}(s, x)\right) d s\right) .
\end{aligned}
$$

Lemma 3.2 Assume that $\varphi$ is borelian and $\|\varphi\|_{0, L^{4}, k}<+\infty$. Then we have

$$
\left|D S_{t} \varphi(x)\right|_{\frac{3}{4}} \leq c e^{c t}\left(1+t^{-7 / 8}\right) \mid\left(|x|_{L^{4}}+1\right)^{k}\|\varphi\|_{0, L^{4}, k}, \quad x \in H, t \geq 0 .
$$

Proof. By approximation, we may assume that $\varphi \in C_{b}(H)$ and use (3.4). Hence, we can write by 
Hölder inequality

$$
\begin{aligned}
& \left|D S_{t} \varphi(x) \cdot h\right| \leq \frac{1}{t}\|\varphi\|_{0, L^{4}, k}\left(\mathbb{E}\left(e^{-K \int_{0}^{t}|X(s, x)|_{L^{4}}^{4} d s}\left(1+|X(t, x)|_{L^{4}}^{k}\right)^{2}\right)\right)^{1 / 2} \\
& \times\left[\left(\mathbb{E}\left(e^{-K \int_{0}^{t}|X(s, x)|_{L^{4}}^{4} d s}\left(\int_{0}^{t}\left(\eta^{h}(s, x), d W(s)\right)\right)^{2}\right)\right)^{1 / 2}\right. \\
& +4 K\|\varphi\|_{0, L^{4}, k}\left(\mathbb{E}\left(e^{-K \int_{0}^{t}|X(s, x)|_{L^{4}}^{4} d s}\left(1+|X(t, x)|_{L^{4}}^{k}\right)^{2}\right)\right)^{1 / 2} \\
& \left.\times\left(\mathbb{E}\left(e^{-K \int_{0}^{t}|X(s, x)|_{L^{4}}^{4} d s}\left(\int_{0}^{t}\left(X^{3}(s, x), \eta^{h}(s, x)\right) d s\right)^{2}\right)\right)^{1 / 2}\right] \\
& =I_{1}+I_{2} .
\end{aligned}
$$

Let us estimate $I_{1}$. Concerning the first factor we have, by Proposition 2.2

$$
\mathbb{E}\left(e^{-K \int_{0}^{t}|X(s, x)|_{L^{4}}^{4} d s}\left(1+|X(t, x)|_{L^{4}}^{k}\right)^{2}\right) \leq c e^{c t}\left(1+|x|_{L^{4}}^{k}\right)^{2} .
$$

Concerning the second factor of $I_{1}$, we use Itô formula as in the proof of Lemma 4.1 in [7] and get

$$
\begin{aligned}
& \mathbb{E}\left(e^{-K \int_{0}^{t}|X(s, x)|_{L^{4}}^{4} d s}\left(\int_{0}^{t}\left(\eta^{h}(s, x), d W(s)\right)\right)^{2}\right) \\
& \leq \mathbb{E}\left(\int_{0}^{t} e^{-K \int_{0}^{s}|X(\tau, x)|_{L^{4}}^{4} d \tau}\left|\eta^{h}(s, x)\right|^{2} d s\right) .
\end{aligned}
$$


Then, using (1.8) and $K a^{8 / 3} \leq K a^{4}+c$, we obtain

$$
\begin{aligned}
& \mathbb{E}\left(e^{-K \int_{0}^{t}|X(s, x)|_{L^{4}}^{4} d s}\left(\int_{0}^{t}\left(\eta^{h}(s, x), d W(s)\right)\right)^{2}\right) \\
& \leq c \mathbb{E}\left(\int_{0}^{t} e^{c s-K \int_{0}^{s}|X(\tau, x)|_{L^{4}}^{\frac{8}{3}} d \tau}\left|\eta^{h}(s, x)\right|_{-3 / 4}^{1 / 2}\left|\eta^{h}(s, x)\right|_{1 / 4}^{3 / 2} d s\right) \\
& \leq c e^{c t} \mathbb{E}\left(\int_{0}^{t} e^{c s-K \int_{0}^{s}|X(\tau, x)|_{L^{4}}^{\frac{8}{3}} d \tau}\left|\eta^{h}(s, x)\right|_{-3 / 4}^{2} d s\right)^{1 / 4} \\
& \times \mathbb{E}\left(\int_{0}^{t} e^{c s-K \int_{0}^{s}|X(\tau, x)|_{L^{4}}^{\frac{8}{3}} d \tau}\left|\eta^{h}(s, x)\right|_{1 / 4}^{2} d s\right)^{3 / 4} \\
& \leq c e^{c t} t^{1 / 4}|h|_{-3 / 4}^{2}
\end{aligned}
$$

by Proposition 3.1. Consequently,

$$
\left|I_{1}\right| \leq c e^{c t} t^{-7 / 8}\|\varphi\|_{0, k}\left(1+|x|_{L^{4}}^{k}\right)|h|_{-3 / 4} .
$$

Let us estimate $I_{2}$. We write

$$
\begin{aligned}
& \mathbb{E}\left(e^{-K \int_{0}^{t}|X(s, x)|_{L^{4}}^{4} d s}\left(\int_{0}^{t}\left(X^{3}(s, x), \eta^{h}(s, x)\right) d s\right)^{2}\right) \\
& \left.\leq \mathbb{E}\left(\left.e^{-K \int_{0}^{t}|X(s, x)|_{L^{4}}^{4} d s}\left(\int_{0}^{t}|X(s, x)|_{L^{4}}^{4} d s\right)^{3 / 2}\left(\int_{0}^{t} \mid \eta^{h}(s, x)\right)\right|_{L^{4}} ^{4} d s\right)^{1 / 2}\right) \\
& \left.\leq c \mathbb{E}\left(\left.e^{-K \int_{0}^{t}|X(s, x)|_{L^{4}}^{4} d s}\left(\int_{0}^{t} \mid \eta^{h}(s, x)\right)\right|_{\frac{1}{4}} ^{4} d s\right)^{1 / 2}\right) \\
& \leq c e^{c t}|h|_{-3 / 4}^{2}
\end{aligned}
$$

thanks again to Proposition 3.1.

Finally, we obtain that

$$
\left|D S_{t} \varphi(x) \cdot h\right| \leq\left. c e^{c t}\left(1+t^{-7 / 8}\right)\left|\left(|x|_{L^{4}}+1\right)^{k}\|\varphi\|_{0, L^{4}, k}\right| h\right|_{-3 / 4}
$$

and the conclusion follows. 
Lemma 3.3 Assume that $\varphi \in C_{b}^{1}(H)$ and that $|D \varphi(x)|_{1} \leq c_{\varphi}$ for all $x \in H$. Then

$$
\left|D S_{t} \varphi(x)\right|_{1} \leq c_{\varphi}+c\|\varphi\|_{0}\left(|x|_{L^{6}}+1\right)^{3} e^{c t} .
$$

Proof. We have in fact, by Propositions 2.2 and 3.1, that

$$
\begin{aligned}
& D S_{t} \varphi(x) \cdot h=\mathbb{E}\left(e^{-K \int_{0}^{t}|X(s, x)|_{L^{4}}^{4} d s} D \varphi(X(t, x)) \cdot \eta^{h}(t, x)\right) \\
& -4 K \mathbb{E}\left(e^{-K \int_{0}^{t}|X(s, x)|_{L^{4}}^{4} d s} \int_{0}^{t}\left(X^{3}(s, x), \eta^{h}(s, x)\right) d s \varphi(X(t, x))\right. \\
& \leq c_{\varphi} \mathbb{E}\left(e^{-K \int_{0}^{t}|X(s, x)|_{L^{4}}^{4} d s}\left|\eta^{h}(t, x)\right|_{-1}\right) \\
& +c\|\varphi\|_{0} \mathbb{E}\left(\sup _{t \in[0, T]}|X(t, x)|_{L^{6}}^{3} \int_{0}^{t} e^{-K \int_{0}^{s}|X(\tau, x)|_{L^{4}}^{4} d \tau}\left|\eta^{h}(s, x)\right| d s\right) \\
& \leq\left(c_{\varphi}|h|_{-1}+c\|\varphi\|_{0}\left(|x|_{L^{6}}+1\right)^{3}|h|_{-1}\right) e^{c t} .
\end{aligned}
$$

\subsection{Estimate of $D P_{t} \varphi(x)$}

Lemma 3.4 Assume that $\varphi \in C_{b}^{1}(H)$ and that $|D \varphi(x)|_{\frac{3}{4}} \leq c_{\varphi}$ for all $x \in H$. Then we have

$$
\left|D P_{t} \varphi(x)\right|_{3 / 4} \leq c_{\varphi}+c\|\varphi\|_{0} e^{c t}\left(|x|_{L^{6}}+1\right)^{4} .
$$

Proof. Let $h \in H$. First, we write that

$$
D P_{t} \varphi(x) \cdot h=D S_{t} \varphi(x) \cdot h+\int_{0}^{t} D S_{t-s}\left(|\cdot|{ }_{L^{4}}^{4} P_{s} \varphi\right)(x) d s .
$$

We estimate the first term using Lemma 3.3. Since

$$
\left\||\cdot|{ }_{L^{4}}^{4} P_{s} \varphi\right\|_{0, L^{4}, 4}=\sup _{x \in H} \frac{|x|_{L^{4}}^{4}\left|P_{s} \varphi(x)\right|}{1+|x|_{L^{4}}^{4}} \leq\|\varphi\|_{0}
$$

we obtain by Lemmas 3.2 that

$$
\begin{aligned}
\left|D P_{t} \varphi(x) \cdot h\right| \leq & \left(c_{\varphi}+c\|\varphi\|_{0}\left(|x|_{L^{6}}+1\right)^{3} e^{c t}\right)|h|_{-1} \\
& +\left.c\|\varphi\|_{0} \int_{0}^{t}\left(1+(t-s)^{-7 / 8}\right)\left|\left(|x|_{L^{4}}+1\right)^{4} e^{c(t-s)} d s\right| h\right|_{-3 / 4} .
\end{aligned}
$$


Therefore

$$
\left|D P_{t} \varphi(x)\right|_{3 / 4} \leq c_{\varphi}+c\|\varphi\|_{0} e^{c t}\left[\left(|x|_{L^{6}}+1\right)^{3}+\left(|x|_{L^{4}}+1\right)^{4}\right] .
$$

and (3.7) follows.

Remark 3.5 Using the same argument as above, we easily prove that if $\varphi$ is in $\mathcal{B}_{b}(H)$ then $P_{t} \varphi$ is in $C^{1}(H)$ for $t>0$ and $\left|D P_{t} \varphi(x)\right|_{3 / 4} \leq c\|\varphi\|_{0} e^{c t}\left(|x|_{L^{4}}+1\right)^{4}\left(1+t^{-7 / 8}\right)$. This clearly implies the strong Feller property of $P_{t}$.

We are now going to improve estimate (3.7).

Proposition 3.6 Assume that $\varphi$ is bounded and $|D \varphi(x)|_{1} \leq c_{\varphi}$. Then we have

$$
\left|D P_{t} \varphi(x)\right|_{1} \leq\left(c_{\varphi}+c\|\varphi\|_{0}\right)\left(|x|_{L^{6}}+1\right)^{8} e^{c t} .
$$

Proof. Since

$$
\begin{aligned}
& D S_{t-s}\left(|x|_{L^{4}}^{4} \varphi(x)\right) \cdot h \\
& =4 \mathbb{E}\left(e^{-K \int_{0}^{t-s}|X(\tau, x)|_{L^{4}}^{4} d \tau}\left(X^{3}(t-s, x), \eta^{h}(t-s, x)\right) \varphi(X(t-s, x))\right) \\
& +\mathbb{E}\left(e^{-K \int_{0}^{t-s}|X(\tau, x)|_{L^{4}}^{4} d \tau}|X(t-s, x)|_{L^{4}}^{4} D \varphi\left(X(t-s, x) \cdot \eta^{h}(t-s, x)\right)\right. \\
& -4 K \mathbb{E}\left(e^{-K \int_{0}^{t-s}|X(\tau, x)|_{L^{4}}^{4} d \tau} \int_{0}^{t-s}\left(X^{3}(\tau, x), \eta^{h}(\tau, x)\right) d \tau|X(t-s, x)|_{L^{4}}^{4} \varphi(X(t-s, x))\right),
\end{aligned}
$$

it follows that

$$
\begin{aligned}
& D P_{t} \varphi(x) \cdot h=D S_{t} \varphi(x) \cdot h \\
& +4 \int_{0}^{t} \mathbb{E}\left(e^{-K \int_{0}^{t-s}|X(\tau, x)|_{L^{4}}^{4} d \tau}\left(X^{3}(t-s, x), \eta^{h}(t-s, x)\right) P_{s} \varphi(X(t-s, x))\right) d s \\
& +\int_{0}^{t} \mathbb{E}\left(e^{-K \int_{0}^{t-s}|X(\tau, x)|_{L^{4}}^{4} d \tau}|X(t-s, x)|_{L^{4}}^{4} D P_{s} \varphi\left(X(t-s, x) \cdot \eta^{h}(t-s, x)\right) d s\right. \\
& -4 K \int_{0}^{t} \mathbb{E}\left(e^{-K \int_{0}^{t-s}|X(\tau, x)|_{L^{4}}^{4} d \tau} \int_{0}^{t-s}\left(X^{3}(\tau, x), \eta^{h}(\tau, x)\right) d \tau|X(t-s, x)|_{L^{4}}^{4} P_{s} \varphi(X(t-s, x))\right) d s \\
& =J_{1}+J_{2}+J_{3}+J_{4} .
\end{aligned}
$$

By (3.6) we have

$$
\left|D S_{t} \varphi(x)\right|_{1} \leq c_{\varphi}+c\|\varphi\|_{0}\left(|x|_{L^{6}}+1\right)^{3} e^{c t} .
$$


Concerning $J_{2}$ we have

$$
\begin{aligned}
& \left|J_{2}\right| \leq 4\|\varphi\|_{0} \mathbb{E}\left(\sup _{t \in[0, T]}|X(t, x)|_{L^{6}}^{3} \int_{0}^{t} e^{-K \int_{0}^{t-s}|X(\tau, x)|_{L^{4}}^{4} d \tau}\left|\eta^{h}(t-s, x)\right| d s\right) \\
& \leq\|\varphi\|_{0}\left(|x|_{L^{6}}+1\right)^{3}\left(\mathbb{E}\left(\int_{0}^{t} e^{-K \int_{0}^{t-s}|X(\tau, x)|_{L^{4}}^{4} d \tau}\left|\eta^{h}(t-s, x)\right|^{2} d s\right)^{2}\right)^{1 / 2} \\
& \leq c\|\varphi\|_{0}\left(|x|_{L^{6}}+1\right)^{3}|h|_{-1} e^{c t}
\end{aligned}
$$

by Propositions 2.2 and 3.1 with $\alpha=-1$.

Concerning $J_{3}$ we have, taking into account Lemma 3.4 and that $|\cdot|_{-\frac{3}{4}} \leq c|\cdot|_{0}$,

$|J|_{3}$

$$
\begin{aligned}
& \leq \mathbb{E}\left(\int_{0}^{t} e^{-K \int_{0}^{t-s}|X(\tau, x)|_{L^{4}}^{4} d \tau}|X(t-s, x)|_{L^{4}}^{4}\left(c_{\varphi}+c\|\varphi\|_{0}\left(|X(t-s, x)|_{L^{6}}+1\right)^{4}\right)\left|\eta^{h}(t-s, x)\right|_{-\frac{3}{4}} e^{c(t-s)} d s\right) \\
& \leq c \mathbb{E}\left(\int_{0}^{t} e^{-K \int_{0}^{t-s}|X(\tau, x)|_{L^{4}}^{4} d \tau}|X(t-s, x)|_{L^{4}}^{4}\left(c_{\varphi}+c\|\varphi\|_{0}\left(|X(t-s, x)|_{L^{6}}+1\right)^{4}\right)\left|\eta^{h}(t-s, x)\right|_{0} e^{c(t-s)} d s\right) \\
& \leq\left(c_{\varphi}+c\|\varphi\|_{0}\right)\left(|x|_{L^{6}}+1\right)^{8}|h|_{-1} e^{c t},
\end{aligned}
$$

by Proposition 3.1.

The last term is treated as the second and can by majorized by

$$
\left|J_{4}\right| \leq c\|\varphi\|_{0}\left(|x|_{L^{6}}+1\right)^{3}\left(|x|_{L^{4}}+1\right)^{4}|h|_{-1} e^{c t} .
$$

We deduce

$$
\left|D P_{t} \varphi(x) \cdot h\right| \leq\left(c_{\varphi}+c\|\varphi\|_{0}\right)\left(|x|_{L^{6}}+1\right)^{8}|h|_{-1} e^{c t}
$$

and the conclusion follows.

\section{Study of the Kolmogorov operator}

\subsection{Essential $m$-dissipativity of $N_{0}$}

We prove that the infinitesimal generator $N_{2}$ of the transition semigroup in $L^{2}(H, \nu)$ is $m$-dissipative and is the closure of the differential operator $N_{0}$ defined by

$$
N_{0} \varphi=\frac{1}{2} \operatorname{Tr} D^{2} \varphi+(A x+b(x), D \varphi), \quad \varphi \in \mathcal{E}_{A}(H) .
$$


We note that, by using the Itô formula, it is easy to check that $N_{2}$ is an extension of $N_{0}$. Consequently, $N_{0}$ is closable and we have only to prove that its closure $\overline{N_{0}}$ coincides with $N_{2}$

To this purpose, we shall proceed as in [1], by introducing an approximating operator

$$
N_{n} \varphi=\frac{1}{2} \operatorname{Tr} D^{2} \varphi+\left(A x+b_{n}(x), D \varphi\right), \quad \varphi \in \mathcal{E}_{A}(H)
$$

where $b_{n}$ are regular approximations of $b$. We can use Galerkin approximations for instance. In this case, $b_{n}=P_{n} b\left(P_{n} \cdot\right)$ where $P_{n}$ is the eigenprojector corresponding to the $n$ first eigenvalues of $A$. Then we consider the equation

$$
\lambda \varphi_{n}-N_{n} \varphi_{n}=f
$$

where $\lambda>0$ and $f \in \mathcal{E}_{A}(H)$, whose solution is given by

$$
\varphi_{n}(x)=\int_{0}^{+\infty} e^{-\lambda t} D P_{t}^{n} f(x) d t
$$

where $P_{t}^{n}$ is the transition semigroup relative to the approximated problem

$$
\left\{\begin{array}{l}
d X_{n}=\left(A X_{n}+b_{n}\left(X_{n}\right)\right) d t+d W(t) \\
X_{n}(0)=x
\end{array}\right.
$$

It can be shown that $\varphi_{n}$ belongs to the closure $\overline{N_{0}}$ of $N_{0}$ and that

$$
\lambda \varphi_{n}(x)-\overline{N_{0}} \varphi_{n}(x)=f(x)+\left(b_{n}(x)-b(x), D \varphi_{n}(x)\right) .
$$

The following result is the key argument to prove the result.

Lemma 4.1 For $\lambda>c$ we have for any $p \geq 1$

$$
\lim _{n \rightarrow \infty}\left(b_{n}(x)-b(x), D \varphi_{n}(x)\right)=0 \quad \text { in } L^{p}(H, \nu) .
$$

Lemma 4.1 implies that the range of $\lambda-\overline{N_{0}}$ is dense in $L^{p}(H, \nu)$ and then the conclusion follows from the Lumer-Phillips theorem, [20]. In fact, we also obtain that the generator in $L^{p}(H, \nu)$ is $m$-dissipative for any $p \geq 1$.

To prove the Lemma, we note that, since $b_{n}$ is the Galerkin projection of $b$, we know that $\left(b_{n}(x)-b(x), D \varphi_{n}(x)\right) \rightarrow 0, \nu$ almost surely and $\left|b_{n}(x)-b(x)\right|_{-1} \leq|b(x)|_{-1}$. Thus it suffices to prove an estimate on

$$
\left.\int_{H}|b(x)|_{-1}^{p} \mid D \varphi_{n}\right)\left.\right|_{1} ^{p} d \nu
$$

for any $p \geq 1$ independently of $n$. Then, we conclude thanks uniform integrability. 
Note that all estimates in the previous sections can be proved on the approximated semigroup $P_{t}^{n}$ and with constant independent of $n$. By (4.2) we have that

$$
D \varphi_{n}(x) \cdot h=\int_{0}^{\infty} e^{-\lambda t} D P_{t}^{n} f(x) \cdot h d t, \quad x, h \in H .
$$

Therefore

$$
\left|D \varphi_{n}(x)\right|_{1} \leq \int_{0}^{\infty} e^{-\lambda t}\left|D P_{t}^{n} f(x)\right|_{1} d t .
$$

Since $f \in \mathcal{E}_{A}(H)$ we have $|D f(x)|_{1} \leq c_{f}$ and so, by Proposition 3.6 it follows that

$$
\begin{aligned}
\left|D \varphi_{n}(x)\right|_{1} & \leq \int_{0}^{\infty} e^{-(\lambda-c) t}\left(c_{f}+c\|f\|_{0}\right)\left(1+|x|_{L^{6}}\right)^{8} d t \\
& =\frac{c_{f}+c\|f\|_{0}}{\lambda-c}\left(1+|x|_{L^{6}}\right)^{8} .
\end{aligned}
$$

Consequently,

$$
|b(x)|_{-1}\left|D \varphi_{n}(x)\right|_{1} \leq c|x|_{L^{4}}^{2}\left|D \varphi_{n}(x)\right|_{1} \leq \frac{\left(c_{f}+c\|f\|_{0}\right)}{\lambda-c}\left(|x|_{L^{6}}+1\right)^{8}|x|_{L^{4}}^{2}
$$

and, by Proposition 2.3 with $p=4$ and $p=6$, we obtain the required estimate. The proof is complete.

\subsection{The integration by parts formula}

Integrating with respect to $\nu$ the obvious identity

$$
N_{0}\left(\varphi^{2}\right)=2 \varphi N_{0} \varphi+\left|Q^{1 / 2} D \varphi\right|^{2}, \quad \varphi \in \mathcal{E}_{A}(H)
$$

and taking into account that $\int_{H} N_{0}\left(\varphi^{2}\right) d \nu=0$ by the invariance of $\nu$, we find that

$$
\int_{H} N_{0} \varphi \varphi d \nu=-\frac{1}{2} \int_{H}|D \varphi|^{2} d \nu
$$

In order to extend (4.5) to all functions of $D\left(N_{2}\right)$, we first define the Sobolev space $W^{1,2}(H, \mu)$. For this we shall show that the derivative operator

$$
D: \mathcal{E}_{A}(H) \subset L^{2}(H, \nu) \rightarrow L^{2}(H, \nu ; H), \varphi \rightarrow D \varphi
$$

is closable. We need two lemmas. 
Lemma 4.2 For any $\varphi \in C_{b}^{1}(H)$ we have that

$$
\left|D S_{t} \varphi(x)\right|^{2} \leq c\left(t^{1 / 8}+1\right)\left(P_{t}\left(\varphi^{2}\right)(x)+P_{t}\left(|D \varphi|^{2}\right)(x)\right), \quad x \in H, t \geq 0
$$

and

$$
\left.\int_{H}\left|D S_{t} \varphi(x)\right|^{2} \nu(d x) \leq c\left(t^{1 / 8}+1\right) \int_{H}\left(\varphi^{2}(x)+|D \varphi(x)|^{2}\right)\right) \nu(d x) \quad t \geq 0 .
$$

Proof. Let $h \in H$ and $\varphi \in C_{b}^{1}(H)$, then we have

$$
\begin{aligned}
& D S_{t} \varphi(x) \cdot h=\mathbb{E}\left(e^{-K \int_{0}^{t}|X(s, x)|_{L^{4}}^{4} d s} D \varphi\left(X(t, x) \cdot \eta^{h}(t, x)\right)\right. \\
& -4 K \mathbb{E}\left(e^{-K \int_{0}^{t}|X(s, x)|_{L^{4}}^{4} d s} \varphi(X(t, x)) \int_{0}^{t}\left(X^{3}(s, x), \eta^{h}(s, x)\right) d s\right) \\
& :=I_{1}+I_{2} .
\end{aligned}
$$

Taking into account Proposition 3.1, we get

$$
\begin{aligned}
\left|I_{1}\right| & \leq\left[\mathbb{E}\left(|D \varphi(X(t, x))|^{2}\right)\right]^{1 / 2}\left[\mathbb{E}\left(e^{-2 K \int_{0}^{t}|X(s, x)|_{L^{4}}^{4} d s}\left|\eta^{h}(t, x)\right|^{2}\right)\right]^{1 / 2} \\
& \leq\left[P_{t}\left(|D \varphi|^{2}\right)(x)\right]^{1 / 2}|h|
\end{aligned}
$$

and, by the Sobolev embedding $H^{1 / 4}(0,1) \subset L^{4}(0,1)$ and (1.8),

$$
\begin{aligned}
\left|I_{2}\right| & \left.\leq 4 K \mathbb{E}\left(\left.e^{-K \int_{0}^{t}|X(s, x)|_{L^{4}}^{4} d s}|\varphi(X(t, x))|\left(\int_{0}^{t}|X(s, x)|_{L^{4}}^{4} d s\right)^{3 / 4}\left(\int_{0}^{t} \mid \eta^{h}(s, x)\right)\right|_{L^{4}} ^{4} d s\right)^{1 / 4}\right) \\
& \left.\leq c\left[\mathbb{E}\left(|\varphi(X(t, x))|^{2}\right)\right]^{1 / 2}\left[\left.\mathbb{E}\left(e^{-K \int_{0}^{t}|X(s, x)|_{L^{4}}^{4} d s} \int_{0}^{t} \mid \eta^{h}(s, x)\right)\right|_{L^{4}} ^{4} d s\right)\right]^{1 / 4} \\
& \leq c t^{1 / 8} P_{t}\left(\varphi^{2}\right)(x)|h|
\end{aligned}
$$

and (4.6) follows. Then (4.7) follows integrating with respect to $\nu$ and taking into account the invariance of $\nu$.

The proof of the second lemma is analogous, using formula (3.4) instead of (4.8). So, we shall omit it.

Lemma 4.3 For any $\varphi \in C_{b}(H)$ we have

$$
\left|D S_{t} \varphi(x)\right|^{2} \leq c\left(t^{-1 / 2}+t^{1 / 8}+1\right) P_{t}\left(\varphi^{2}\right)(x) \quad x \in H, t>0
$$


and

$$
\int_{H}\left|D S_{t} \varphi(x)\right|^{2} \nu(d x) \leq c\left(t^{-1 / 2}+t^{1 / 8}+1\right) \int_{H} \varphi^{2}(x) \nu(d x), \quad t>0 .
$$

We can now prove the result

Proposition 4.4 D is closable.

Proof. Let $\left\{\varphi_{n}\right\} \subset \mathcal{E}_{A}(H)$ such that

$$
\varphi_{n} \rightarrow 0 \quad \text { in } L^{2}(H, \nu), \quad D \varphi_{n} \rightarrow F \quad \text { in } L^{2}(H, \nu ; H),
$$

as $n \rightarrow \infty$, where $F \in L^{2}(H, \nu ; H)$. We have to show that $F=0$.

Let $t>0$. Then by (4.10) it follows that

$$
D S_{t} \varphi_{n} \rightarrow 0 \quad \text { in } L^{2}(H, \nu ; H), \text { as } n \rightarrow \infty .
$$

On the other hand by (4.8) it follows that for any $h \in H$,

$$
\begin{aligned}
& \int_{H}\left|\mathbb{E}\left(e^{-K \int_{0}^{t}|X(s, x)|_{L^{4}}^{4} d s} F(X(t, x)) \cdot \eta^{h}(t, x)\right)\right|^{2} d \nu \\
& \leq \int_{H}\left|\mathbb{E}\left(e^{-K \int_{0}^{t}|X(s, x)|_{L^{4}}^{4} d s}\left(F(X(t, x))-D \varphi_{n}(X(t, x))\right) \cdot \eta^{h}(t, x)\right)\right|^{2} d \nu \\
& +c \int_{H}\left|D S_{t} \varphi_{n}(x) \cdot h\right|^{2} d \nu \\
& +c \int_{H}\left|\mathbb{E}\left(e^{-K \int_{0}^{t}|X(s, x)|_{L^{4}}^{4} d s} \varphi_{n}(X(t, x)) \int_{0}^{t}\left(X^{3}(s, x), \eta^{h}(s, x)\right) d s\right)\right|^{2} d \nu .
\end{aligned}
$$

Using Proposition 3.1, (4.11), (4.12) and the invariance of $\nu$, we easily prove that each term on the right hand side converges to zero. Thus, we deduce

$$
\int_{H}\left|\mathbb{E}\left(e^{-K \int_{0}^{t}|X(s, x)|_{L^{4}}^{4} d s} F(X(t, x)) \cdot \eta^{h}(t, x)\right)\right|^{2} d \nu=0
$$

for $t>0$. Then, we write

$$
\begin{aligned}
\int_{H}|\mathbb{E}(F(X(t, x)) \cdot h)| d \nu & \leq \int_{H}\left|\mathbb{E}\left(F(X(t, x)) \cdot\left(e^{-K \int_{0}^{t}|X(s, x)|_{L^{4}}^{4} d s} \eta^{h}(t, x)-h\right)\right)\right| d \nu \\
& \leq\left(\int_{H} \mathbb{E}\left(|F(X(t, x))|^{2}\right) d \nu\right)^{1 / 2}\left(\int_{H} \mathbb{E}\left(\left|e^{-K \int_{0}^{t}|X(s, x)|_{L^{4}}^{4} d s} \eta^{h}(t, x)-h\right|^{2}\right) d \nu\right)^{1 / 2}
\end{aligned}
$$

It follows from the dominated convergence theorem that the right hand side converges to zero as $t \rightarrow 0$. Moreover

$$
\int_{H}|\mathbb{E}(F(X(t, x)) \cdot h)| d \nu \rightarrow \int_{H}|F(x) \cdot h| d \nu
$$


so that we obtain

$$
\int_{H}|F(x) \cdot h| d \nu=0
$$

Therefore, for each $h \in H, F(x) \cdot h=0, \nu$ almost surely. Since $H$ is countable, it follows that $F(x)=0, \nu$ almost surely.

We can now define the space $W^{1,2}(H, \nu)$ as the domain of the closure of $D$ and extend the validity of (4.5).

Proposition 4.5 The following inclusion holds,

$$
D\left(N_{2}\right) \subset W^{1,2}(H, \nu) .
$$

Moreover,

$$
\int_{H} N_{2} \varphi \varphi d \nu=-\frac{1}{2} \int_{H}|D \varphi|^{2} d \nu, \quad \text { for all } \varphi \in D\left(N_{2}\right)
$$

Proof. Let $\varphi \in D\left(N_{2}\right)$. Since $\mathcal{E}_{A}(H)$ is a core for $N_{2}$, there exists a sequence $\left\{\varphi_{n}\right\} \subset \mathcal{E}_{A}(H)$ such that

$$
\varphi_{n} \rightarrow \varphi, \quad N_{0} \varphi_{n} \rightarrow N_{2} \varphi \quad \text { in } L^{2}(H, \nu) .
$$

Now, for any $m, n \in \mathbb{N}$ we find from (4.5),

$$
\int_{H}\left|D\left(\varphi_{m}-\varphi_{n}\right)\right|^{2} d \nu=-2 \int_{H} N_{2}\left(\varphi_{m}-\varphi_{n}\right)\left(\varphi_{m}-\varphi_{n}\right) d \nu
$$

Consequently, the sequence $\left\{\varphi_{n}\right\}$ is Cauchy in $W^{1,2}(H, \nu)$ and the conclusion follows.

Let us now show another useful identity.

Proposition 4.6 Let $T>0$ and $u(t)=P_{t} \varphi$. Then $u \in L^{2}\left(0, T ; W^{1,2}(H, \nu)\right)$ and the following identity holds.

$$
\int_{H}|u(t)|^{2} d \nu+\int_{0}^{t} d s \int_{H}|D u(s)|^{2} d \nu=\int_{H}|\varphi|^{2} d \nu
$$

Proof. Let first $\varphi \in D\left(N_{2}\right)$ and set $u(t)=P_{t} \varphi$. Then, multiplying the identity

$$
\frac{d}{d t} u(t)=N_{2} u(t), \quad t \geq 0
$$

by $u(t)$ and integrating in $H$ with respect to $\nu$ and taking into account (4.14) yields

$$
\frac{1}{2} \frac{d}{d t} \int_{H}|u(t)|^{2} d \nu=\int_{H} N_{2} u(t) u(t)=-\frac{1}{2} \int_{H}|D u(t)|^{2} d \nu .
$$

So, identity (4.15) holds for all $\varphi \in D\left(N_{2}\right)$. 
Let now $\varphi \in L^{2}(H, \nu)$. Since $N_{2}$ is dense in $L^{2}(H, \nu)$ there exists a sequence $\left\{\varphi_{n}\right\} \subset D\left(N_{2}\right)$ such that

$$
\varphi_{n} \rightarrow \varphi, \quad N_{2} \varphi_{n} \rightarrow N_{2} \varphi \quad \text { in } L^{2}(H, \nu) .
$$

Set $u_{n}(t)=P_{t} \varphi_{n}$. Then by (4.14) it follows that

$$
\int_{0}^{T} d s \int_{H}\left|D\left(u_{n}-u_{m}\right)\right|^{2} d \nu \leq \int_{H}\left|\varphi_{n}-\varphi_{m}\right|^{2} d \nu,
$$

so that the sequence $\left\{u_{n}\right\}$ is Cauchy in $L^{2}\left(0, T ; W^{1,2}(H, \nu)\right)$ and the conclusion follows.

The following result is useful to study Poincaré inequality and spectral gap.

Corollary 4.7 Let $\varphi \in L^{2}(H, \nu)$. Then the following identity holds

$$
\int_{H}|\varphi-\bar{\varphi}|^{2} d \nu=\int_{0}^{+\infty}\left\|D P_{s} \varphi\right\|_{L^{2}(H, \nu)}^{2} d s .
$$

where $\bar{\varphi}=\int_{H} \varphi d \nu$.

Proof. Let $\varphi \in L^{2}(H, \nu)$ and set $u(t)=P_{t} \varphi$. Letting $t \rightarrow+\infty$ in (4.15) and taking into account that by the Doob theorem

$$
\lim _{t \rightarrow \infty} P_{t} \varphi=\bar{\varphi} \quad \text { in } L^{2}(H, \nu)
$$

we see that

$$
(\bar{\varphi})^{2}+\int_{0}^{+\infty}\left\|D P_{s} \varphi\right\|_{L^{2}(H, \nu)}^{2} d s=\|\varphi\|^{2}
$$

that is equivalent to $(4.16)$.

\section{References}

[1] V. Barbu, G. Da Prato and A. Debussche The transition semigroup of stochastic Navier-Stokes equations in $2-D$, Infinite Dimensional Analysis, Quantum Probability and related topics, to appear.

[2] L. Bertini, N. Cancrini and G. Jona-Lasinio, The stochastic Burgers equation, Commun. Math. Phys. 165, No.2, 211-232, 1994.

[3] J. M. Bismut, Large deviations and the Malliavin calculus, Birkhäuser, 1984.

[4] C. Cardon-Weber, Large deviations for a Burgers'-type SPDE, Stochastic Processes Appl. 84, No.1, 53-70, 1999.

[5] H. Crauel and F. Flandoli, Attractors for random dynamical systems, Probab. Theory Relat. Fields 100, No.3, 365-393, 1994.

[6] G. Da Prato and A. Debussche, Maximal dissipativity of the Dirichlet operator corresponding to the Burgers equation, CMS Conference Proceedings, vol. 28, Canadian Mathematical Society, 145-170, 2000. 
[7] G. Da Prato and A. Debussche, Ergodicity for the 3D stochastic Navier-Stokes equation, Journal de Mathématiques Pures et Appliquées, 82, 877-947, 2003.

[8] G. Da Prato, A. Debussche and R. Temam, Stochastic Burgers equation, Nonlinear Differential Equations and Applications, 389-402, 1994.

[9] G. Da Prato and D. Gatarek Stochastic Burgers equation with correlated noise, Stochastics Stochastics Rep. 52, No.1-2, 29-41, 1995.

[10] G. Da Prato and M. Röckner, Weak solutions to stochastic porous media equations, Jounal of Evolution Equations (to appear).

[11] G. Da Prato and J. Zabczyk, Stochastic equations in infinite dimensions, Encyclopedia of Mathematics and its Applications, Cambridge University Press, 1992.

[12] G. Da Prato and J. Zabczyk, Second order partial differential equations in Hilbert spaces, London Mathematical Society, Lecture Note Series. 293. Cambridge University Press.

[13] G. Da Prato and J. Zabczyk, Differentiability of the Feynman-Kac semigroup and a control application, Rend. Mat. Accad. Lincei, 8, 183-188, 1997.

[14] A. Debussche Hausdorff dimension of a random invariant set, Journal de Mathématiques Pures et Appliquées, 77, p.967-988, 1998.

[15] K. D. Elworthy, Stochastic flows on Riemannian manifolds, Diffusion processes and related problems in analysis, Vol. II, M. A. Pinsky and V. Wihstutz (editors), 33-72, Birkhäuser, 1992.

[16] M. Fukushima, Y. Oshima and M. Takeda Dirichlet forms and symmetric Markov processes, de Gruyter, Berlin, 1994.

[17] I. Gyongy, Existence and uniqueness results for semilinear stochastic partial differential equations in Hilbert space, Stochastic Processes and their Applications, 73, 271-299, 1998.

[18] V. Liskevitch and M. Röckner Strong uniqueness for a class of infinite dimensional Dirichlet operators and application to stochastic quantization, Ann. Scuola Norm. Sup. Pisa Cl. Sci. (4),Vol. XXVII, pp. 69-91, 1998.

[19] J. Leon, D. Nualart and R. Pettersson, The stochastic Burgers equation: Finite moments and smoothness of the density, Infin. Dimens. Anal. Quantum Probab. Relat. Top. 3, No.3, 363-385, 2000.

[20] G. Lumer and R. S. Phillips, Dissipative operators in a Banach space, Pac. J. Math., 11, 679-698, 1961.

[21] M. Röckner, $L^{p}$-analysis of finite and infinite dimensional diffusion, in Stochastic PDE's and Kolmogorov equations in infinite dimensions. Lectures given at the 2nd session of the Centro Internazionale Matematico Estivo (CIME) held in Cetraro, Italy, August 24 - September 1, 1998. Berlin: Springer. Lect. Notes Math. 1715, 65-116, 1999

[22] M. Röckner et Z. Sobol Kolmogorov equations in infinite dimensions: well-posedness and regularity of solutions, with applications to stochastic generalized Burgers equations, to appear.

[23] W. Stannat, The theory of generalized Dirichlet forms and its applications in Analysis and Stochastics, Memoirs AMS, 678, 1999. 